\title{
AOR
}

Selected Papers of \#AolR2020:

The 22nd Annual Conference of the

Association of Internet Researchers Virtual Event / 13-16 Oct 2021

\section{THE PROS AND PERILS OF INTERDEPENDENCE: FEMINIST ORGANIZING IN ONLINE GAME FORUMS}

\author{
Amanda Cote \\ University of Oregon

\section{Introduction}

Although many women play video games, the medium's association with masculinity remains strong. Female gamers are often harassed as outsiders, which can drive them to hide their presence and make it difficult to connect with one another (Brehm, 2013; Cote, 2020; Eklund, 2011; Fox \& Tang, 2017; Gray, 2014). Lacking a strong support system, and in the face of toxicity, many women drop out of gaming over time. This loss suggests a need for greater interdependence between female gamers, to provide interpersonal support and to develop collective responses to gaming's inequalities.

Feminism is meant to be collective, rather than individualist, as only group action can resolve structural problems (Banet-Weiser, 2018), and research suggests that targeted internet communities, such as Facebook groups or discussion boards, could promote feminist networking, consciousness-raising, and action. Clark-Parsons (2018) and Pruchniewska (2019) found that women-only Facebook groups advanced a range of feminist practices, from implicitly supporting workplace equality to explicitly organizing politically. Rentschler and Thrift (2015) argue that sharing feminist memes can help build larger networked publics. Female gamers could thus benefit from online groups.

But online communities may have limits. The internet is often considered a masculinized or identity-free space (Herring et al., 2002), meaning feminist discussions can face disruption or backlash. Braithwaite (2014), for instance, found that online forum participants who raised concerns about sexism in World of Warcraft were seen as "killjoys" and consequently faced high levels of vitriol. Explicitly feminist forums provide space in which such conversations are accepted, but they can draw trolls who disrupt the groups' functioning or harass its members (e.g. Herring et al., 2002; Rentschler \& Thrift, 2015; Shaw, 2013). Groups that police their boundaries to avoid these problems may then implicitly prioritize some participants-i.e. straight, white, cisgender womenover others (Clark-Parsons, 2018; Pruchniewska, 2019). 
Therefore, while online spaces could help female gamers build interdependencies and raise feminist consciousness, we must assess if/how they accomplish these goals and for whom. This paper begins to explore these issues through a comparison of two online gaming forums-one general and one female-specific. It asks:

1) How (if at all) do female-oriented gaming forums differ from general gaming forums in their discussion of feminist issues?

2) What opportunities does this offer for consciousness-raising and collective action?

\section{Methods}

While the overall project aims to address online communities writ large, this paper starts with text-based discussion forums, which provide a consistent format for analysis. Further, it focuses specifically on how forum participants post about harassment and negativity. Players of all kinds face harassment in games, making it a general topic of concern. However, in-game toxicity is also a feminist issue, as the level/type of harassment directed at female gamers often differs from that directed at male gamers (Brehm, 2013; Cote, 2020; Fox \& Tang, 2017). Conversations about harassment allow us to assess if, when, and how it's discussed as a general vs. a gendered problem, as well as if discourses differ by forum. This can provide insight into opportunities for consciousness-raising and collective action.

Following the example of Bergstrom (2021), I identified two forums of interest and searched them for the terms "toxic," "harassment," and "bullying," importing the results into the qualitative analysis software NVivo. Initial analysis focused on the top 100 posts per search term (600 total), coding each for the following variables:

1) relevance (e.g. a thread on toxic players would be relevant; a thread on a toxic waste environment in a game would not)

2) number of likes

3) number of comments

4) overall topic (e.g. venting, sexism, news, meme, etc.; up to three labels/post)

Given the sensitive nature of conversations about harassment, the searchability of online forums, and in line with ethical guidelines for internet research (e.g. Franzke et al., 2020), I am choosing not to identify the specific forums or quote from them directly. However, both forums are large (over 100,000 members each) and active, providing a up-to-date, inside look at gaming communities.

\section{Preliminary Findings}

Quantitatively, the female-oriented community produced more relevant posts (276/300 vs. 184/300) and more consistent engagement via likes and comments. It also showed a higher like/comment ratio, which could indicate greater support for those sharing their experiences or starting discussions. The lower like/comment ratio in the general community reveals more disagreement; highly commented posts in this forum are almost as likely to be disliked as they are to be liked. The forums also showed thematic 
differences. Female-oriented community posts largely focused on sharing personal experiences with negativity, explicitly calling out sexism, and discussing coping mechanisms for avoiding or managing harassment. In contrast, the general community took a more abstract approach to harassment and negativity, sharing news articles and memes or engaging in general discussions.

Moving forward, I will perform a critical discourse analysis (CDA) of comments on these top posts, to assess how forum participants discuss these topics in depth. This preliminary investigation, however, expands upon research that suggests female players are already independent activists to address what opportunities they could gain from greater interdependence. It suggests that there are differences between online communities and that the female-oriented forum's higher support may lay a foundation for collective networking, consciousness-raising, and action, such as through the sharing of coping mechanisms.

\section{References}

Banet-Weiser, S. (2018). Empowered: Popular Feminism and Popular Misogyny. Duke University Press.

Bergstrom, K. (2021). Anti-social social gaming: Community conflict in a Facebook game. Critical Studies in Media Communication, 1-14.

Braithwaite, A. (2014). 'Seriously, get out': Feminists on the forums and the War(craft) on women. New Media \& Society, 16(5), 703-718.

Brehm, A. L. (2013). Navigating the feminine in massively multiplayer online games: Gender in World of Warcraft. Frontiers in Psychology, 4(DEC), 903.

Clark-Parsons, R. (2018). Building a digital Girl Army: The cultivation of feminist safe spaces online. New Media \& Society, 20(6), 2125-2144.

Cote, A. C. (2020). Gaming Sexism: Gender and Identity in the Era of Casual Video Games. New York University Press.

Eklund, L. (2011). Doing gender in cyberspace: The performance of gender by female World of Warcraft players. Convergence, 17(3), 323-342.

Fox, J., \& Tang, W. Y. (2017). Women's experiences with general and sexual harassment in online video games: Rumination, organizational responsiveness, withdrawal, and coping strategies. New Media \& Society, 19(8), 1290-1307.

Franzke, A. S., Bechmann, A., Zimmer, M., Ess, C. M., \& Association of Internet Researchers. (2020). Internet Research: Ethical Guidelines 3.0. https://aoir.org/reports/ethics3.pdf

Gray, K. L. (2014). Race, gender, and deviance in Xbox Live: Theoretical perspectives from the virtual margins. Elsevier. 
Herring, S., Job-Sluder, K., Scheckler, R., \& Barab, S. (2002). Searching for safety online: Managing "trolling" in a feminist forum. Information Society, 18(5), 371-384.

Pruchniewska, U. (2019). "A group that's just women for women": Feminist affordances of private Facebook groups for professionals. New Media and Society, 21(6), 13621379.

Rentschler, C. A., \& Thrift, S. C. (2015). Doing feminism in the network: Networked laughter and the 'Binders Full of Women' meme. Feminist Theory, 16(3), 329-359.

Shaw, F. (2013). Still "Searching for Safety Online": collective strategies and discursive resistance to trolling and harassment in a feminist network. The Fibreculture Journal, 22, 93-108. 